TRANSACTIONS OF THE

AMERICAN MATHEMATICAL SOCIETY

Volume 362, Number 6, June 2010, Pages 3095-3106

S 0002-9947(09)04904-6

Article electronically published on August 13, 2009

\title{
ON SLICING INVARIANTS OF KNOTS
}

\author{
BRENDAN OWENS
}

\begin{abstract}
The slicing number of a knot, $u_{s}(K)$, is the minimum number of crossing changes required to convert $K$ to a slice knot. This invariant is bounded above by the unknotting number and below by the slice genus $g_{s}(K)$. We show that for many knots, previous bounds on the unknotting number obtained by Ozsváth and Szabó and by the author in fact give bounds on the slicing number. Livingston defined another invariant $U_{s}(K)$, which takes into account signs of crossings changed to get a slice knot and which is bounded above by the slicing number and below by the slice genus. We exhibit an infinite family of knots $K_{n}$ with slice genus $n$ and Livingston invariant greater than $n$. Our bounds are based on restrictions (using Donaldson's diagonalisation theorem or Heegaard Floer homology) on the intersection forms of four-manifolds bounded by the double branched cover of a knot.
\end{abstract}

\section{INTRODUCTION}

The unknotting number of a knot is the minimum number of crossing changes required to convert it to an unknot. Ozsváth and Szabó used Heegaard Floer theory to provide a powerful obstruction to a knot having unknotting number one 22. This obstruction was generalised in [17] to higher unknotting numbers. In this paper we show that similar techniques yield information about the number of crossing changes required to convert to a slice knot.

The slice genus $g_{s}(K)$ of a knot $K$ in the three-sphere is the minimum genus of a connected oriented smoothly properly embedded surface in the four-ball with boundary $K$. A knot is called a slice if $g_{s}(K)=0$. Given any diagram $D$ for a knot $K$, a new knot may be obtained by changing one or more crossings of $D$. The slicing number $u_{s}(K)$ is the minimum number of crossing changes required to obtain a slice knot, where the minimum is taken over all diagrams for $K$. A "movie" of a sequence of crossing changes represents an immersed annulus in $S^{3} \times[0,1]$ with a singularity for each crossing change. A neighbourhood of each singular point may be removed and replaced with an annulus; if the last frame of the movie is a slice knot, then the corresponding boundary component of the resulting embedded surface may be capped off with a disk, yielding a surface in $B^{4}$ with genus $u_{s}(K)$ and boundary $K$.

Recall that crossings in a knot diagram may be given a sign as in Figure 1 (independent of the choice of orientation of the knot). Suppose that $K$ may be sliced (converted to a slice knot) by changing $p$ positive and $n$ negative crossings (in some diagram). Form the immersed annulus in $S^{3} \times[0,1]$ as before. The sign

Received by the editors April 11, 2008.

2000 Mathematics Subject Classification. Primary 57M25.

The author was supported in part by NSF grant DMS-0604876. 


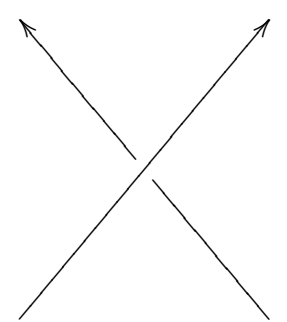

Positive

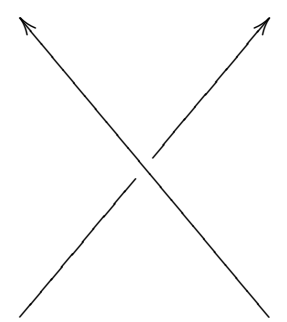

Negative

FIGURE 1. Signed crossings in a knot diagram.

of each self-intersection of this annulus agrees with the sign of the corresponding crossing in the changed diagram. Take two self-intersections of opposite sign, and in each case remove a disk neighbourhood of the singularity from just one of the intersecting sheets and connect the boundary components by a tube. This leads to an oriented surface in $B^{4}$ with genus $\max (p, n)$. Livingston defined the following slicing invariant:

$$
U_{s}(K)=\min (\max (p, n)),
$$

where the minimum is taken over all diagrams for $K$ and over all sets of crossing changes in a diagram which give a slice knot.

From the preceding discussion we see that

$$
g_{s}(K) \leq U_{s}(K) \leq u_{s}(K) .
$$

Livingston showed in [12] that the two-bridge knot $S(15,4)$, also known as $7_{4}$, has $u_{s}=2$ and $g_{s}=1$, thus giving a negative answer to a question of Askitas [16. (Murakami and Yasuhara showed in [14] that $8_{16}$ has $u_{s}=2$ and $g_{s}=1$. Their proof is based on a four-manifold bounded by the double-branched cover of the knot. We take a similar approach here.) Livingston also asked whether in fact $U_{s}$ is always equal to the slice genus, and suggested that $7_{4}$ may be a counterexample.

Let $\sigma(K)$ denote the signature of a knot $K$. It is shown in [3, Proposition 2.1] (also [24, Theorem 5.1]) that if $K^{\prime}$ is obtained from $K$ by changing a positive crossing, then

$$
\sigma\left(K^{\prime}\right) \in\{\sigma(K), \sigma(K)+2\} ;
$$

similarly if $K^{\prime}$ is obtained from $K$ by changing a negative crossing, then

$$
\sigma\left(K^{\prime}\right) \in\{\sigma(K), \sigma(K)-2\} .
$$

Now suppose that $K$ may be sliced by changing $p$ positive and $n$ negative crossings (in some diagram). Since a slice knot has zero signature, it follows that a bound for $n$ is given by

$$
n \geq \sigma(K) / 2 .
$$

In this paper we give an obstruction to the equality in (1).

Let $\Sigma(K)$ denote the double cover of $S^{3}$ branched along $K$, and suppose that crossing changes in some diagram for $K$ result in a slice knot $J$. It follows from "Montesinos' trick" ([13, or see [17]) that $\Sigma(K)$ is given by Dehn surgery on some framed link in $\Sigma(J)$ with half-integral framing coefficients. 
Definition 1. An integer-valued symmetric bilinear form $Q$ on a free abelian group of rank $2 r$ is said to be of half-integer surgery type if it admits a basis $\left\{x_{1}, \ldots, x_{r}, y_{1}, \ldots, y_{r}\right\}$ with

$$
\begin{aligned}
& Q\left(x_{i}, x_{j}\right)=2 \delta_{i j}, \\
& Q\left(x_{i}, y_{j}\right)=\delta_{i j} .
\end{aligned}
$$

Examples. The positive-definite rank 2 unimodular form is of half-integer surgery type since it may be represented by the matrix $\left(\begin{array}{ll}2 & 1 \\ 1 & 1\end{array}\right)$. The form represented by the matrix $\left(\begin{array}{ll}4 & 1 \\ 1 & 4\end{array}\right)$ is not of half-integer surgery type since it has no vectors of square 2 (the vector $x$ with coefficients $(a, b)$ has square $4 a^{2}+2 a b+4 b^{2}=$ $(a+b)^{2}+3\left(a^{2}+b^{2}\right)$; since $a$ and $b$ are integers one cannot solve $\left.Q(x, x)=2\right)$.

Converting the half-integer surgery description above to integer surgery in the standard way gives a cobordism $W$ from $\Sigma(J)$ to $\Sigma(K)$ whose intersection form $Q_{W}$ is of half-integer surgery type. Since $J$ is slice, $\Sigma(J)$ bounds a rational homology ball $B$. Joining $B$ to $W$ along $\Sigma(J)$ gives a smooth closed four-manifold $X$ bounded by $\Sigma(K)$. The second Betti number of $X$ is twice the number of crossing changes used to get from $K$ to $J$.

Suppose now that $K$ is converted to $J$ by changing $p$ positive and $n$ negative crossings, with $n=\sigma(K) / 2$. Then $K$ bounds a disk in $B^{4} \#^{p+n} \mathbb{C P}^{2}$. As in [3] (see also [17]) we may take the double cover $X^{\prime}$ of the blown-up four-ball branched along this disk. It follows from a theorem of Cochran and Lickorish [3, Theorem 3.7] that $X^{\prime}$ is positive-definite with $b_{2}\left(X^{\prime}\right)=2(p+n)$. The following theorem is based on the idea that in fact $X$ is diffeomorphic to $X^{\prime}$.

Theorem 2. Suppose that a knot $K$ can be converted to a slice knot by changing $p$ positive and $n$ negative crossings, with $n=\sigma(K) / 2$. Then the branched double cover $\Sigma(K)$ bounds a positive-definite smooth four-manifold $X$ with $b_{2}(X)=$ $2(p+n)$ whose intersection form $Q_{X}$ is of half-integer surgery type, with exactly $n$ of $Q_{X}\left(x_{1}, x_{1}\right), \ldots, Q_{X}\left(x_{p+n}, x_{p+n}\right)$ even, and $\operatorname{det} Q_{X}$ divides $\operatorname{det} K$ with quotient a square.

For knots whose determinant is square-free, it follows that the first two parts of Ozsváth and Szabó's obstruction to unknotting number one [22, Theorem 1.1] (without the symmetry condition) in fact give an obstruction to $u_{s}(K)=1$.

Corollary 3. The knots

$$
\begin{aligned}
& 7_{4}, 8_{16}, 9_{5}, 9_{15}, 9_{17}, 9_{31}, 10_{19}, 10_{20}, 10_{24}, 10_{36}, 10_{68}, 10_{69}, 10_{86}, \\
& 10_{97}, 10_{105}, 10_{109}, 10_{116}, 10_{121}, 10_{122}, 10_{144}, 10_{163}, 10_{165}
\end{aligned}
$$

have slice genus 1 and slicing number 2 .

(Note that as mentioned above this was shown for $7_{4}$ in [12] and for $8_{16}$ in [14]. The slice genus information in Corollaries 3 and 4 is taken from [1.)

Furthermore, the obstruction given in [17, Theorem 5] to unknotting a knot by changing $p$ positive and $n=\sigma(K) / 2$ negative crossings is in fact an obstruction to slicing, provided again that $\operatorname{det} K$ is square-free. 
Corollary 4. The knots

$$
9_{10}, 9_{13}, 9_{38}, 10_{53}, 10_{101}, 10_{120}
$$

have slice genus 2 and slicing number 3 .

The 11-crossing two-bridge knot $S(51,35)$ (Dowker-Thistlethwaite name 11a365) has slice genus 3 and slicing number 4.

It may also be shown that for some of the knots in Corollaries 3 and 4 , Livingston's invariant $U_{s}$ is not equal to the slice genus. The knot $7_{4}$ is such an example (as Livingston suggested in 12]), and in fact we find that it is the first member of an infinite family of such examples.

Corollary 5. For each positive integer $n$, there exists a two-bridge knot $K_{n}$ with signature $2 n$ and slice genus $n$ which cannot be sliced by changing $n$ negative crossings and any number of positive crossings; hence $U_{s}\left(K_{n}\right)>n$.

\section{Proof of Theorem 2}

In this section we prove our main result.

Recall that a positive-definite integer-valued symmetric bilinear form $Q$ on a free abelian group $A$ gives an integer lattice $L$ in Euclidean space on tensoring with $\mathbb{R}$. We say a lattice $L$ in $\mathbb{R}^{n}$ is of half-integer surgery type if the corresponding form $Q$ is of the form given in Definition 1. Also, a matrix representative for $Q$ is referred to as a Gram matrix for $L$. For convenience we will frequently denote $Q(x, y)$ by $x \cdot y$, and $Q(x, x)$ by $x^{2}$.

The proof of Theorem 2 consists of a topological and an algebraic step. Following 17. we show using careful analysis of Montesinos' trick that, under the hypotheses of the theorem, $\Sigma(K)$ bounds a positive-definite manifold $X$ and that the intersection pairing of $X$ is of half-integer surgery type when restricted to some finite index sublattice. We then show that if a lattice $M$ has an odd index sublattice $L$ of half-integer surgery type, then in fact $M$ is of half-integer surgery type.

We begin with a couple of lemmas.

Lemma 2.1. Let $Q$ be a form of half-integer surgery type, with $m_{i}=Q\left(y_{i}, y_{i}\right)$. Then

$$
\operatorname{det} Q \equiv \prod_{i=1}^{r}\left(2 m_{i}-1\right) \quad(\bmod 4) .
$$

Proof. This follows from the discussion after Lemma 2.2 in [17.

Lemma 2.2. Let $Q$ be a block matrix of $r \times r$ blocks of the form $\left(\begin{array}{cc}2 I & * \\ * & *\end{array}\right)$ which is congruent modulo 2 to $\left(\begin{array}{cc}2 I & I \\ I & X\end{array}\right)$. Then there exists $P=\left(\begin{array}{cc}I & * \\ 0 & R\end{array}\right) \in G L(2 r, \mathbb{Z})$ with

$$
P^{T} Q P=\left(\begin{array}{cc}
2 I & I \\
I & X^{\prime}
\end{array}\right)
$$

and $X^{\prime} \equiv X(\bmod 2)$.

Proof. Let $Q$ be the Gram matrix of a lattice with basis $x_{1}, \ldots, x_{r}, z_{1}, \ldots, z_{r}$. By successively adding multiples of $x_{i}$ to each of $z_{1}, \ldots, z_{r}$ we get a new basis $x_{1}, \ldots, x_{r}$, $y_{1}, \ldots, y_{r}$ with $x_{i} \cdot y_{j}=\delta_{i j}$; since $x_{i}$ has an even square, this preserves parities on the diagonal. 
The following was originally proved by Ozsváth and Szabó 22 in the case $p+n=$ 1 and $J$ is the unknot.

Proposition 2.3. Suppose that a knot $K$ can be converted to a slice knot $J$ by changing $p$ positive and $n$ negative crossings, with $n=\sigma(K) / 2$. Then the branched double cover $\Sigma(K)$ bounds a positive-definite four-manifold $X$ with $b_{2}(X)=$ $2(p+n)$. The lattice $\left(H_{2}(X ; \mathbb{Z}), Q_{X}\right)$ contains a finite index sublattice of halfinteger type, which has a basis as in Definition 11 with exactly $p$ elements of odd square.

Proof. We adapt the proof of [17, Lemma 3.2]. By Montesinos' lemma ([13, or see [17, Lemma 3.1]), $\Sigma(J)$ is the result of surgery on some link $L$ in $S^{3}$ with halfinteger framing coefficients. Convert to integer surgery (see [6] or [17, Lemma 2.2]): the framing $(2 m-1) / 2$ on any component is replaced with $m$, and a small 2 -framed meridian is added to each component. Let $Q_{J}$ be the resulting linking matrix of half-integer type. We may assume (after possibly adding a $-1 / 2$ framed unknot to $L)$ that $\operatorname{det} Q_{J}$ is positive. This integer framed link is a Kirby diagram for a fourmanifold $X_{J}$ obtained by attaching a two-handle to the four-ball along each framed link component, i.e. a four-dimensional two-handlebody. The two-handlebody $X_{J}$ has boundary $\Sigma(J)$ and an intersection form represented by $Q_{J}$.

Note that since $J$ is slice it has signature zero and determinant $\operatorname{det} J=\operatorname{det} Q_{J}=$ $k^{2}$ for some odd integer $k$. Suppose that $K_{-}$is a knot of signature 2 which may be converted to $J$ by changing a single negative crossing $c$. Then $\Sigma(K)$ is the result of surgery on $L \cup C$ for some $\operatorname{knot} C$ in $S^{3}$, with framing $(2 m-1) / 2$ on $C$. Let $K_{0}$ be the result of taking the oriented resolution of the crossing $c$. Then as in [17, Lemma 3.2] we have that $\Sigma\left(K_{0}\right)$ is surgery on $L \cup C$ with framing $m$ on $C$. Converting to integer surgery we find that $\Sigma\left(K_{-}\right), \Sigma\left(K_{0}\right)$ are given by integer surgeries with linking matrices

$$
Q_{-}=\left(\begin{array}{ccc}
2 & 1 & 0 \\
1 & m & * \\
0 & * & Q_{J}
\end{array}\right), \quad Q_{0}=\left(\begin{array}{cc}
m & * \\
* & Q_{J}
\end{array}\right) .
$$

Let $\Delta_{L}(t)$ denote the Conway-normalised Alexander polynomial of a link $L$ (see for example [9, Chapter 8]). For a knot $K$, the sign of the Alexander polynomial at -1 is given by

$$
\Delta_{K}(-1)=(-1)^{\sigma(K) / 2} \operatorname{det} K .
$$

(This may be seen by diagonalising the symmetrised Seifert form as in [15, Proof of Theorem 5.6].) This shows that

$$
\Delta_{J}(-1)=k^{2}, \Delta_{K_{-}}(-1)=-\left|\operatorname{det} Q_{-}\right|=-\left|2 \operatorname{det} Q_{0}-k^{2}\right| .
$$

The skein relation for the Alexander polynomial then yields

$$
k^{2}+\left|2 \operatorname{det} Q_{0}-k^{2}\right|=2\left|\operatorname{det} Q_{0}\right|,
$$

from which we conclude that $\operatorname{det} Q_{-}=2 \operatorname{det} Q_{0}-k^{2}$ is positive. Now using Lemma 2.1 we have

$$
2 m-1 \equiv \operatorname{det} Q_{-}=\operatorname{det} K_{-} \equiv 3 \quad(\bmod 4),
$$

and thus $m$ is even. (The last congruence is due to Murasugi [15]: $\operatorname{det} K \equiv \sigma(K)+1$ $(\bmod 4)$.) 
Now suppose $K_{+}$is a knot of signature 0 which may be converted to $J$ by changing a positive crossing. Again $\Sigma(K)$ is obtained by half-integer surgery on $L \cup C$ for some knot $C$ in $S^{3}$, with framing $(2 m-1) / 2$ on $C$. Let $Q_{+}$denote the linking matrix after converting to integer surgery. A similar argument as above (see 17. for the case where $J$ is the unknot) shows that $\operatorname{det} Q_{+}>0$ and $m$ is odd.

Let $c_{1}, \ldots, c_{p+n}$ be the set of crossings ( $p$ positive, $n$ negative) in some chosen diagram of $K$ that we change to convert to $J$. Then $\Sigma(K)$ is Dehn surgery on the link $L \cup C_{1} \cup \cdots \cup C_{p+n}$ with half-integer framing coefficients. Each $C_{i}$ corresponds to a crossing $c_{i}$. Dehn surgery on $L$ union a sublink of $C_{1} \cup \cdots \cup C_{p+n}$ gives the double branched cover of a knot which is obtained from $K$ by changing a subset of the crossings $c_{1}, \ldots, c_{p+n}$. In particular, surgery on the knot $L \cup C_{i}$ yields the double branched cover of the knot $K_{i}^{\prime}$ which is obtained from $K$ by changing all of the crossings except $c_{i}$. By the condition $n=\sigma(K) / 2$ the knot signature changes every time a negative crossing is changed and remains constant when a positive crossing changes. It follows from the discussion above applied to $K_{i}^{\prime}$ that the framing on each $C_{i}$ is of the form $\left(2 m_{i}-1\right) / 2$ and exactly those $m_{i}$ which correspond to changing negative crossings of $K$ are even.

Denote by $X_{K}$ the two-handlebody with boundary $\Sigma(K)$ that results from converting to integer surgery (i.e. surgery on the link $L \cup C_{1} \cup \cdots \cup C_{p+n}$, followed by surgery on a 2 -framed meridian of each component). Then $X_{K}$ has intersection form of half-integer surgery type; moreover we can view $X_{K}$ as the union of $X_{J}$ and a surgery cobordism $W$ along the common boundary $\Sigma(J)$. We will show by an induction argument that this cobordism is positive-definite.

Let $K_{j}$ denote the knot obtained from $K$ by changing crossings $c_{j+1}, \ldots, c_{p+n}$, and let $Q_{j}$ be the linking matrix of half-integer type obtained by converting the corresponding Dehn surgery diagram of $\Sigma\left(K_{j}\right)$ to integer type.

Suppose that $\operatorname{det} Q_{j-1}$ is positive and hence equal to $\operatorname{det} K_{j-1}$. We have

$$
Q_{j}=\left(\begin{array}{ccc}
2 & 1 & 0 \\
1 & m_{j} & * \\
0 & * & Q_{j-1}
\end{array}\right),
$$

and by Lemma 2.1

$$
\operatorname{det} Q_{j} \equiv\left(2 m_{j}-1\right) \operatorname{det} Q_{j-1} \quad(\bmod 4) .
$$

If $c_{j}$ is a positive crossing, then $m_{j}$ is odd, and so

$$
\operatorname{det} Q_{j} \equiv \operatorname{det} Q_{j-1} \quad(\bmod 4) \text {. }
$$

On the other hand the signature of $K_{j-1}$ is equal to that of $K_{j}$, and so

$$
\operatorname{det} K_{j} \equiv \operatorname{det} K_{j-1} \quad(\bmod 4)
$$

Comparing (21) and (3) we see that $\operatorname{det} Q_{j}$ is congruent modulo 4 to its absolute value. Since it is an odd number it must be positive.

On the other hand if $c_{j}$ is negative we find both congruences (2) and (3) do not hold, and again it follows that $\operatorname{det} Q_{j}$ is positive.

By induction we see that $Q_{J}=Q_{0}, Q_{1}, \ldots, Q_{p+n}$ all have positive determinants. Thus the surgery cobordism $W$ is built by attaching $2(p+n)$ two-handles to the two-handlebody $X_{J}$, and before and after each attachment we have a four-manifold 
whose intersection pairing has positive determinant. It follows that $W$ is positivedefinite.

We claim $\left(H_{2}(W ; \mathbb{Z}), Q_{W}\right)$ contains a finite index sublattice with a basis as in Definition 1 with exactly $p$ elements of odd square. Suppose that $L$ has $r$ components, so that $b_{2}\left(X_{K}\right)=2(p+n+r)$. Let $\left\{x_{i}, y_{i}\right\}$ be a basis for $H_{2}\left(X_{K} ; \mathbb{Z}\right)$ as in Definition 1, chosen so that $\left\{x_{i}, y_{i}\right\}_{i>p+n}$ is a basis for the sublattice $H_{2}\left(X_{J} ; \mathbb{Z}\right)$. For $i \leq p+n$, take $y_{i}$ to be the class corresponding to the two-handle attached along $C_{i}$, and let $x_{i}$ be that class corresponding to the two-handle attached along the meridian of $C_{i}$. Note that each $x_{i}$ is contained in $H_{2}(W ; \mathbb{Z})$. The rough idea is to form a sublattice by projecting the span of $\left\{x_{i}, y_{i}\right\}_{i \leq p+n}$ orthogonally to $H_{2}(W ; \mathbb{Z})$. Of course we cannot quite do this; however, since $H_{1}(\Sigma(J) ; \mathbb{Z})$ has order $k^{2}$, we may write

$$
k^{2} y_{i}=z_{i}+w_{i}, \quad i=1, \ldots, p+n,
$$

with $z_{i} \in H_{2}(W ; \mathbb{Z})$ and $w_{i} \in H_{2}\left(X_{J} ; \mathbb{Z}\right)$. We claim that the self-intersection of $z_{i}$ has the same parity as that of $y_{i}$. To see this note that for each $j>p+n$, $y_{i}$ is orthogonal to $x_{j}$, and hence so is $w_{i}$. It follows that $w_{i}$ is in the span of $\left\{x_{i}\right\}_{i>p+n}$ and so has even self-intersection. Thus we have a full rank sublattice of $\left(H_{2}(W ; \mathbb{Z}), Q_{W}\right)$ with basis $\left\{x_{i}, z_{i}\right\}_{i \leq p+n}$. By Lemma 2.2, this sublattice has a basis as in Definition 1 with exactly $p$ elements of odd square.

Form the manifold $X$ with one boundary component by capping off the $\Sigma(J)$ end of $W$ with the rational ball $B$ given as the double branched cover of $B^{4}$ along a slice disk bounded by $J[2]$. Then $\left(H_{2}(W ; \mathbb{Z}), Q_{W}\right)$ is a finite index sublattice of $\left(H_{2}(X ; \mathbb{Z}), Q_{X}\right)$, and therefore so is $\operatorname{Span}\left(\left\{x_{i}, z_{i}\right\}_{i \leq p+n}\right)$.

Proposition 2.4. Suppose $M$ is a positive-definite integer lattice of rank $2 r$ and $L$ is a sublattice of $M$ of odd index $l$. If $L$ is of half-integer type, then so is $M$. Moreover, the number of elements of a basis of $M$ as in Definition 1 with odd square is the same as that for $L$.

This will follow from the next two lemmas, the first of which is standard.

Lemma 2.5. The natural map from $G L(n, \mathbb{Z})$ to $G L(n, \mathbb{Z} / 2)$ is onto for any $n$.

Proof. Use induction on $n$. Suppose that $R \in M(n, \mathbb{Z})$ has odd determinant. The cofactor expansion across the first row yields

$$
\operatorname{det} R=r_{11} R_{11}+r_{12} R_{12}+\cdots+r_{1 n} R_{1 n} .
$$

Since the determinant is odd, so is at least one $r_{1 j} R_{1 j}$. By induction we may choose $\tilde{R} \equiv R(\bmod 2)$ with $\tilde{R}_{1 j}=1$, and then adjust the value of $r_{1 j}$ to get $\operatorname{det} \tilde{R}=1$.

Lemma 2.6. Suppose $M$ is a positive-definite integer lattice of rank $2 r$, and that $L$ is a lattice of half-integer type which is a sublattice of $M$ of odd index l. Let $x_{1}, \ldots, x_{r}, y_{1}, \ldots, y_{r}$ be a basis for $L$ as in Definition 1, and let $Q_{L}$ be the Gram matrix of $L$ in this basis. Then $x_{1}, \ldots, x_{r}$ may be extended to a basis $x_{1}, \ldots, x_{r}, z_{1}, \ldots, z_{r}$ for $M$ with

$$
Q_{M} \equiv Q_{L} \quad(\bmod 2)
$$

Proof. Let $m_{i}=y_{i} \cdot y_{i}$. In the given basis $Q_{L}$ is in block form $\left(\begin{array}{cc}2 I & I \\ I & X\end{array}\right)$, with $\operatorname{Diag}(X)=\left(m_{1}, \ldots, m_{r}\right)$. By Theorem 6 in Chapter 1, Section 3 of [7], there exists a basis $a_{1}, \ldots, a_{r}, z_{1}, \ldots, z_{r}$ of $M$ with $x_{i} \in \operatorname{Span}_{\mathbb{Z}}\left\{a_{1}, \ldots, a_{i}\right\}$. A simple induction 
argument using the fact that $x_{i} \cdot y_{j}=\delta_{i j}$ shows that in fact we may take $a_{i}=x_{i}$ for $i=1, \ldots, r$.

Let $P \in M(2 r, \mathbb{Z})$ be the matrix whose $i$ th column is the coefficient vector of the $i$ th basis vector of $L$ in the basis $x_{1}, \ldots, x_{r}, z_{1}, \ldots, z_{r}$. Then $P$ is in block form $\left(\begin{array}{cc}I & * \\ 0 & R\end{array}\right)$, and

$$
Q_{L}=P^{T} Q_{M} P
$$

Since $\operatorname{det} Q_{L}=l^{2} \operatorname{det} Q_{M}$ we have $\operatorname{det} R=l$ is odd. By Lemma 2.5 we may choose $\tilde{R} \in G L(r, \mathbb{Z})$ with $\tilde{R} \equiv R(\bmod 2)$. Applying the transition matrix $\tilde{P}=\left(\begin{array}{ll}I & * \\ 0 & \tilde{R}\end{array}\right)$ to $M$ yields the required basis.

Proof of Proposition 2.4. Let $x_{1}, \ldots, x_{r}, z_{1}, \ldots, z_{r}$ be the basis of $M$ given by Lemma 2.6, in which

$$
Q_{M}=\left(\begin{array}{cc}
2 I & * \\
* & *
\end{array}\right) \equiv Q_{L}=\left(\begin{array}{cc}
2 I & I \\
I & X
\end{array}\right) \quad(\bmod 2)
$$

The proposition now follows from Lemma 2.2 .

Proof of Theorem 2, Let $X$ be the four-manifold bounded by $\Sigma(K)$ given by Proposition 2.3. A standard argument using the long exact homology sequence of the pair $(X, \Sigma(K))$ shows that $\operatorname{det} Q_{X}$ divides $\operatorname{det} K$ with its quotient a square (see for example [18, Lemma 2.1]). The determinant of a knot is always odd. It follows that $Q_{X}$ has odd order and the sublattice given by Proposition 2.3 has odd index in $\left(H_{2}(X ; \mathbb{Z}), Q_{X}\right)$. Theorem 2 now follows immediately from Proposition 2.4 .

\section{EXAMPLES}

Theorem 2 tells us that to show that a knot $K$ cannot be converted to a slice knot by changing $p$ positive and $n=\sigma(K) / 2$ negative crossings, we must show that its double branched cover cannot bound a four-manifold with an intersection form with certain properties.

We will make use of two very effective gauge-theoretic obstructions to a rational homology three-sphere $Y$ bounding a positive-definite form $Q$. On the one hand, Ozsváth and Szabó define a function

$$
d: \operatorname{Spin}^{c}(Y) \rightarrow \mathbb{Q}
$$

coming from the absolute grading in Heegaard Floer homology, and they show that for each $\operatorname{spin}^{c}$ structure $\mathfrak{s}$ on a positive-definite four manifold bounded by $Y$ the following must hold:

$$
\begin{aligned}
c_{1}(\mathfrak{s})^{2}-b_{2}(X) & \geq 4 d\left(\left.\mathfrak{s}\right|_{Y}\right) \\
\text { and } \quad c_{1}(\mathfrak{s})^{2}-b_{2}(X) & \equiv 4 d\left(\left.\mathfrak{s}\right|_{Y}\right) \quad(\bmod 2) .
\end{aligned}
$$

The left-hand side depends on the intersection form of $X$.

To determine if a given knot $K$ may be sliced by changing $p$ positive and $n=$ $\sigma(K) / 2$ negative crossings, we carry out the following steps:

1. Compute $d: \operatorname{Spin}^{c}(\Sigma(K)) \rightarrow \mathbb{Q}$.

2. Find a complete finite set of representatives $Q_{1}, \ldots, Q_{m}$ of forms of rank $2(p+n)$ satisfying the conclusion of Theorem 2 . 
3. Check using (4), (5) whether $\Sigma(K)$ is obstructed from bounding each of $Q_{1}$, $\ldots, Q_{m}$.

Details on Heegaard Floer theory and the $d$ invariant may be found in [20, 21, 22. For a summary of how this theory may be used in our context see [17.

Another approach to understanding the set of positive-definite forms that a threemanifold $Y$ may bound is to make use of Donaldson's diagonalisation theorem [4]. This approach works well for Seifert fibred rational homology spheres, and especially for lens spaces (see e.g. [10, 11, 19]). Knowledge of a particular negative-definite four-manifold $X_{1}$ bounded by $Y$ constrains the intersection form of a positivedefinite $X_{2}$ with the same boundary, since $X=X_{2} \cup-X_{1}$ is a closed positivedefinite manifold with $\left(H_{2}(X ; \mathbb{Z}), Q_{X}\right) \cong \mathbb{Z}^{m}$ for some $m$. We will illustrate this technique in Subsection 3.3 .

The slicing number of a knot is the same as that for its reflection. We assume in what follows that all knots have nonnegative signature. (This distinguishes between the knot and its reflection unless the signature is zero.)

3.1. Knots with slice genus one. For a knot with $\sigma(K)=2$ and $u_{s}(K)=1$, it follows from inequality (11) and Theorem 2 that $\Sigma(K)$ bounds a four-manifold whose intersection form is represented by the matrix

$$
\left(\begin{array}{cc}
m & 1 \\
1 & 2
\end{array}\right)
$$

with $\operatorname{det} K=(2 m-1) t^{2}$ for some integer $t$.

For a knot with $\sigma(K)=0$ and $u_{s}(K)=1$, we find that either $\Sigma(K)$ or $-\Sigma(K)$ must bound such a positive-definite four-manifold.

The knots listed in Corollary 3 have square-free determinant. For each of them, Ozsváth and Szabó have shown in 22, using (4), (5), that $\pm \Sigma(K)$ cannot bound

$$
\left(\begin{array}{cc}
\frac{\operatorname{det} K+1}{2} & 1 \\
1 & 2
\end{array}\right) \text {. }
$$

It is also known in each case that the knot can be unknotted with two crossing changes. We conclude that $u_{s}(K)=2$.

Remark 3.1. Each of the knots $10_{29}, 10_{40}, 10_{65}, 10_{67}, 10_{89}, 10_{106}$, and $10_{108}$ has signature 2 and $\operatorname{det} K=s t^{2}$ for some $t>1$. In each case Ozsváth and Szabó have shown $\Sigma(K)$ cannot bound $\left(\begin{array}{cc}\frac{\operatorname{det} K+1}{2} & 1 \\ 1 & 2\end{array}\right)$, and hence $K$ has unknotting number two.

However we find in each case $\Sigma(K)$ is not obstructed from bounding $\left(\begin{array}{cc}\frac{s+1}{2} & 1 \\ 1 & 2\end{array}\right)$.

3.2. Knots with slice genus two or three. We now consider the knots in Corollary 4 Each of $9_{10}, 9_{13}, 9_{38}, 10_{53}, 10_{101}, 10_{120}$ has signature 4 and slice genus 2 . In 17. we have shown, using (4), (5), that in each case $\Sigma(K)$ cannot bound a positivedefinite form $Q$ as in Definition 1 with rank 4 , $\operatorname{det} Q=\operatorname{det} K$ and $Q\left(x_{i}, x_{i}\right)$ even. Since the knots have square-free determinant, it follows from Theorem 2 that they cannot be sliced with two crossing changes. Each can be unknotted with three crossing changes, and so in each case $u_{s}(K)=3$.

Similarly $K=11 a 365$ is shown in [17] to have unknotting number 4 , and since its determinant is square-free the same argument shows that it has $u_{s}(K)=4$. 
3.3. Knots with large slice genus. In this subsection we prove Corollary 5 . We define $K_{n}$ to be the 4-plat closure of the four-string braid $\left(\sigma_{1}^{4} \sigma_{2}^{4}\right)^{n}$, as illustrated in Figure 2 for $n=2$. For $n=1$ this is the knot $7_{4}=S(15,11)$ shown by Lickorish to have unknotting number 2 and by Livingston to have slicing number 2 [8, 12].

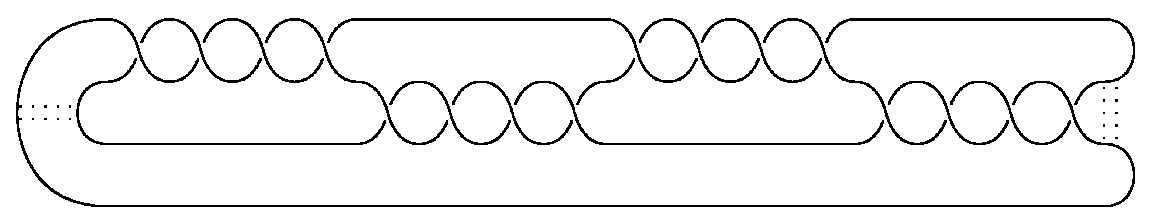

Figure 2. The knot $K_{2}$. The two pairs of dashed arcs indicate where to attach ribbons to go from $K_{n}$ to $K_{n-1}$.

As illustrated in the diagram, two oriented ribbon moves convert $K_{n}$ to $K_{n-1}$. Since $K_{0}$ is the unknot this shows that the slice genus of $K_{n}$ is at most $n$. The signature of $K_{n}$ may be shown (see below) to be $2 n$. We conclude that

$$
g_{s}\left(K_{n}\right)=n \text {. }
$$

Let $P\left(a_{1}, a_{2}, \ldots, a_{m}\right)$ denote the plumbing of disk bundles over two-spheres corresponding to the linear graph with $m$ vertices, where the $i$ th vertex has weight $a_{i}$. The double cover of $S^{3}$ branched along $K_{n}$ is the boundary of $P(4,4, \ldots, 4)$. Let $Q_{n}$ denote the rank $2 n$ intersection form of this plumbing, and let $L_{n}$ denote the associated lattice in $\mathbb{R}^{2 n}$. The following sequence of blow-ups and blow-downs exhibits $\Sigma\left(K_{n}\right)$ as the boundary of a negative-definite plumbing:

$$
\begin{aligned}
\Sigma\left(K_{n}\right) & \cong \partial P(4,4, \ldots, 4) \\
& \cong \partial P(-1,2,-1,2, \ldots,-1,2,-1) \\
& \cong \partial P(-2,-1,1,-2,-1,1, \ldots,-2,-1,1,-1) \\
& \cong \partial P(-2,-2,-3,-2,-3,-2, \ldots,-3,-2,-3,-2,-2)
\end{aligned}
$$

(We note that the above shows that $K_{n}$ may also be represented by the alternating diagram which is the closure of the braid

$$
\left(\sigma_{1}^{-1} \sigma_{2}^{2}\right)^{2 n} \sigma_{1}^{-1} \text {. }
$$

Using the formula of Gordon and Litherland [5] it is easy to compute the signature from this diagram.)

Let $X_{n}^{\prime}$ denote the positive-definite plumbing $P(2,2,3,2,3,2, \ldots, 3,2,2)$ with boundary $-\Sigma\left(K_{n}\right)$. Let $Q_{n}^{\prime}$ denote its intersection form and let $L_{n}^{\prime}$ denote the associated lattice. Note that $L_{n}^{\prime}$ has dimension $2 n+3$ : there are $n$ vertices with weight 3 and $n+3$ with weight 2 .

Lemma 3.2. Suppose $\Sigma\left(K_{n}\right)$ is given as the boundary of a smooth four-manifold $X$ with positive-definite intersection form $Q_{X}$. Then $\left(H_{2}(X ; \mathbb{Z}), Q_{X}\right)$ embeds as a finite index sublattice of $L_{n} \oplus \mathbb{Z}^{k}$ for some $k \geq 0$. 
Proof. Gluing $X$ to $X_{n}^{\prime}$ along their boundary gives a closed positive-definite manifold. It follows from Donaldson's theorem that the lattice $L_{n}^{\prime}$ embeds as a sublattice of $\mathbb{Z}^{m}$ with the lattice $\left(H_{2}(X ; \mathbb{Z}), Q_{x}\right)$ contained in its orthogonal complement.

Let $e_{1}, \ldots, e_{m}$ be an orthonormal basis of $\mathbb{Z}^{m}$. Up to automorphism of $\mathbb{Z}^{m}$ there is a unique way to embed $L_{n}^{\prime}$ : the first vertex vector must map to $e_{1}+e_{2}$, the second to $e_{2}+e_{3}$, the third to $e_{3}+e_{4}+e_{5}$, the fourth to $e_{5}+e_{6}$, and so on. Thus the image of $L_{n}^{\prime}$ is contained in a $\mathbb{Z}^{3 n+4}$ sublattice of $\mathbb{Z}^{m}$. An easy calculation shows that the orthogonal complement of $L_{n}^{\prime}$ in $\mathbb{Z}^{3 n+4}$ is spanned by the vectors $e_{1}-e_{2}+e_{3}-e_{4}$, $e_{4}-e_{5}+e_{6}-e_{7}, \ldots, e_{3 n+1}-e_{3 n+2}+e_{3 n+3}-e_{3 n+4}$. These span a copy of $L_{n}$, from which the conclusion follows.

Lemma 3.3. For any $n \geq 1, k \geq 0$, the lattice $L_{n} \oplus \mathbb{Z}^{k}$ does not admit any finite index sublattices of half-integer surgery type.

Proof. For $k=0$ this is immediate, since $L_{n}$ has no nonzero vectors of square less than 4 . If $k>0$ let $e_{1}, \ldots, e_{k}$ be an orthonormal basis of $\mathbb{Z}^{k}$, and suppose we have a sublattice of $L_{n} \oplus \mathbb{Z}^{k}$ of half-integer surgery type with basis $\left\{x_{i}, y_{i}\right\}$ as in Definition 1. Up to an automorphism of $L_{n} \oplus \mathbb{Z}^{k}$ we have $x_{1}=e_{1}+e_{2}$. Then $x_{2}$ is orthogonal to $x_{1}$. We cannot have $x_{2}=e_{1}-e_{2}$, since $y_{1}$ pairs evenly with $x_{2}$ and oddly with $x_{1}$. Thus up to automorphism, $x_{2}=e_{3}+e_{4}$. It follows that any sublattice of $L_{n} \oplus \mathbb{Z}^{k}$ of half-integer surgery type has rank at most $k$.

Corollary 5 now follows from Lemmas 3.2 and 3.3 and Theorem 2

Remark 3.4. Livingston conjectured in 12 that the difference $U_{s}-g_{s}$ can be arbitrarily large. It is possible to unknot $K_{n}$ by changing $2 n$ positive crossings in the diagram as in Figure 2, In the absence of any further evidence it is tempting to conjecture that for these knots $U_{s}-g_{s}=n$. In any case this would seem to be a good candidate with which to attempt to verify Livingston's conjecture.

Remark 3.5. The trace of a homotopy from a knot $K$ to a slice knot $J$ is an immersed annulus in $S^{3} \times I$. Capping this off with a slice disk yields an immersed disk $D$ in $B^{4}$ bounded by $K$. If $J$ is obtained from $K$ by changing $p$ positive and $n$ negative crossings, then the resulting disk $D$ has $p$ negative self-intersections and $n$ positive self-intersections. (This is why changing a positive crossing is often referred to as a "negative crossing change".) Instead of considering crossing changes, one may ask whether a knot $K$ bounds an immersed disk in $B^{4}$ with a prescribed number of self-intersections, or with prescribed numbers of self-intersections of each sign.

Rudolph has shown in 23. that the minimal number of self-intersections in a ribbon immersed disk bounded by $K$ is equal to the minimal number of crossing changes to get from $K$ to a ribbon knot. (Here a ribbon surface in $B^{4}$ is one on which the radial distance function has no maxima, and a ribbon knot is a knot which bounds an embedded ribbon disk.) Knowing whether a result analagous to Rudolph's for nonribbon disks and slice knots holds would be very interesting. It is to be expected that the conclusion of Theorem 2 holds under the weaker hypothesis that $K$ bounds an immersed disk with $p$ negative, $n=\sigma(K) / 2$ positive self-intersections. The expected proof would generalise that of [14] and also make use of [3, Theorem 3.7].

\section{ACKNOWLEDGEMENTS}

It is a pleasure to thank András Stipsicz and Tom Mark for helpful conversations. 


\section{REFERENCES}

[1] J. C. Cha \& C. Livingston, Table of knot invariants, http://www.indiana.edu/ knotinfo.

[2] A. J. Casson \& C. McA. Gordon, On slice knots in dimension three, Algebraic and geometric topology, Part 2, Proc. Sympos. Pure Math. XXXII, Amer. Math. Soc., 1978, 39-53. MR.520521 (81g:57003)

[3] T. D. Cochran \& W. B. R. Lickorish, Unknotting information from 4-manifolds, Trans. Amer. Math. Soc. 297 1986, 125-142. MR849471 (87i:57003)

[4] S. K. Donaldson, An application of gauge theory to four-dimensional topology, J. Diff. Geom. 18 1983, 279-315. MR.710056 (85c:57015)

[5] C. McA. Gordon \& R. A. Litherland, On the signature of a link, Invent. Math. 471978 , 53-69. MR0500905 (58:18407)

[6] R. E. Gompf \& A. I. Stipsicz, 4-manifolds and Kirby calculus, Graduate Studies in Math. 20, Amer. Math. Soc., 1999. MR1707327 (2000h:57038)

[7] P. M. Gruber \& C. G. Lekkerkerker, Geometry of numbers, 2nd ed., North-Holland Mathematical Library 37, North-Holland, 1987. MR893813 (88j:11034)

[8] W. B. R. Lickorish, The unknotting number of a classical knot, Contemp. Math. 44 1985, 117-121. MR813107 (87a:57012)

[9] W. B. R. Lickorish, An introduction to knot theory, Graduate Texts in Math. 175, Springer, 1997. MR 1472978 (98f:57015)

[10] P. Lisca, Symplectic fillings and positive scalar curvature, Geometry and Topology 2 1998, 103-116. MR1633282 (99f:57038)

[11] P. Lisca, Lens spaces, rational balls and the ribbon conjecture, Geometry and Topology 11 2007, 429-472. MR2302495 (2008a:57008)

[12] C. Livingston, The slicing number of a knot, Algebraic and Geometric Topology 22002 , 1051-1060. MR.1936979(2003m:57018)

[13] J. M. Montesinos, Variedades de Seifert que son cubiertas ciclicas ramificados de dos hojas, Bol. Soc. Mat. Mexicana 18 1973, 1-32. MR0341467(49:6218)

[14] H. Murakami \& A. Yasuhara, Four-genus and four-dimensional clasp number of a knot, Proc. Amer. Math. Soc. 128 2000, 3693-3699. MR1690998 (2001b:57020)

[15] K. Murasugi, On a certain numerical invariant of link types, Trans. Amer. Math. Soc. 117 1965, 387-422. MR.0171275 (30:1506)

[16] T. Ohtsuki, Problems on invariants of knots and 3-manifolds, Invariants of knots and 3manifolds (Kyoto 2001), Geometry and Topology Monographs 4 2002, 377-572. MR 2065029 (2005c:57014)

[17] B. Owens, Unknotting information from Heegaard Floer homology, Advances in Mathematics, 217 (2008), pages 2353-2376. MR2388097

[18] B. Owens \& S. Strle, Rational homology spheres and the four-ball genus of knots, Advances in Mathematics 200, 2006, 196-216. MR2199633 (2006m:57016)

[19] B. Owens \& S. Strle, A characterisation of the $n\langle 1\rangle \oplus\langle 3\rangle$ form and applications to rational homology spheres, Math. Res. Lett. 13, 2006, 259-271. MR2231116 (2007j:57036)

[20] P. Ozsváth \& Z. Szabó, Absolutely graded Floer homologies and intersection forms for four-manifolds with boundary, Advances in Mathematics 173 2003, 179-261. MR 1957829 (2003m:57066)

[21] P. Ozsváth \& Z. Szabó, On the Floer homology of plumbed three-manifolds, Geometry and Topology 7 2003, 225-254. MR1988285 (2004f:57040)

[22] P. Ozsváth \& Z. Szabó, Knots with unknotting number one and Heegaard Floer homology, Topology 44 2005, 705-745. MR2136532 (2005m:57045)

[23] L. Rudolph, Braided surfaces and Seifert ribbons for closed braids, Comment. Math. Helv. 58 1983, 1-37. MR699004 (84j:57006)

[24] A. Stoimenow, Polynomial values, the linking form and unknotting numbers, Math. Res. Lett. 11 2004, 755-769. MR2106240 (2005j:57022)

Department of Mathematics, Louisiana State University, Baton Rouge, Louisiana 70803

Current address: Department of Mathematics, University of Glasgow, Glasgow, G12 8QW, United Kingdom 